\title{
Towards Real-Time Mode Content Characterization of Multimode Fibers
}

\author{
D.R. Gray, S.R. Sandoghchi, N.V. Wheeler, G.T. Jasion, J.P. Wooler, M.N. Petrovich, F. Poletti and \\ D.J. Richardson
}

Optoelectronics Research Centre, University of Southampton, Southampton, SO171BJ, UK, D.R.Gray@soton.ac.uk

\begin{abstract}
We present a new $S^{2}$-based technique with potential for near real-time (1s) characterization of the modal-content of multimode fibers. We also demonstrate the identification and removal of measurement artifacts originating from reflections from optical components.
\end{abstract}

\section{Introduction}

Multimode (MM) fibers have become a topic of interest recently due to their use in modedivision multiplexing for increased data capacity transmission, and in high power laser systems to reduce non-linear effects. Therefore, there is a need to develop MM fiber modal characterization methods that are fast and stable, and that have both high resolution and a high dynamic range. In recent years, interferometric-based methods have shown the most promise due to their high accuracy and the important advantage that no prior knowledge of the fiber is required. Within this category the current forerunner is Spatial and Spectral imaging ${ }^{1}\left(S^{2}\right)$, using a tunable laser source (TLS) and CCD camera to capture the variation of spatial amplitude over frequency, Fig.1a. It measures the differential group delay (DGD), the relative modal power (commonly termed $\mathrm{MPI}=10 \log _{10}\left[\mathrm{LP}_{\mathrm{HOM}} / \mathrm{LP}_{01}\right]$ ) and provides images of both the intensity and the phase of the higher order modes relative to the fundamental mode. The maximum DGD that can be measured is currently limited by the spectral resolution and linewidth of the TLS used; to date 4000ps is the largest delay reported ${ }^{2}$. However, the $S^{2}$ technique has two key limitations. It typically takes minutes for a single measurement and spurious interference peaks are often observed that can limit the dynamic range.

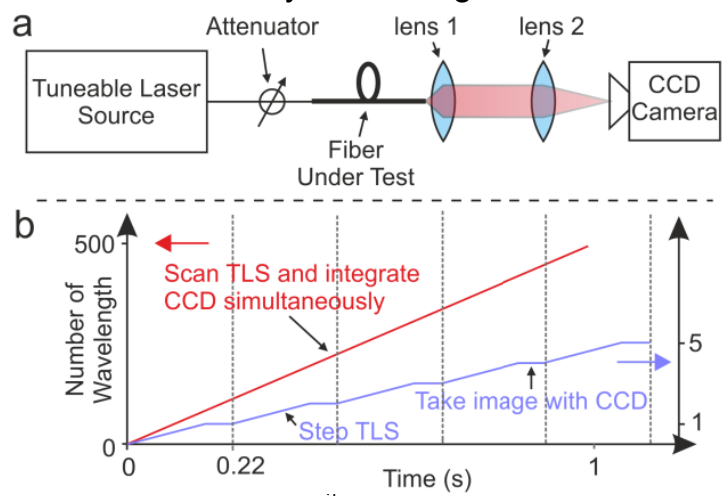

Fig. 1: a) Schematic of $S^{2}$ b) Capture method comparison: standard $S^{2}$ (blue) vs. new method (red).
The sampling speed of the technique is limited to a few hundred wavelengths in a few minutes, which can require an hour of measurement for the highest resolution spectrograms so far reported ${ }^{3}$. Furthermore, any variation in the detected power over time will be measured as a change in power over frequency and thus also add noise in the DGD graph. Spurious interference peaks are also often observed and cannot easily be removed, and can add power to the reported higher order mode content if the interference peaks overlap. Spurious peaks can be split into two categories based on their origin; the first result from interference between higher order modes ${ }^{4}$ when the dominant fundamental mode requirement is broken, and the second are systematic peaks caused by the equipment used. As $S^{2}$ measures the change in power as a function of wavelength, any power change over wavelength not caused by the fiber under test (FUT) will also manifest itself as noise in the DGD data. These spurious delays are often misinterpreted, ignored or cause an increase in the measurement noise floor.

This paper, to the best of our knowledge, reports the first $S^{2}$-based system with a near real-time single measurement capacity (1s), providing an equivalent 110 -fold improvement in measurement capture speed relative to the current state-of-the-art ${ }^{5}$. Furthermore, we identify the physical origins of spurious systematic double reflection peaks in our data and present a solution for their removal.

\section{Near Real-time measurement}

The conventional $S^{2}$ measurement tunes the TLS to one wavelength, which is then launched into the FUT and the output imaged by a CCD camera. The TLS is re-tuned and another image is then taken, Fig. 1b (blue). This process is repeated until images at all the required wavelengths have been measured. The fastest reported system to date took 100 measurements 

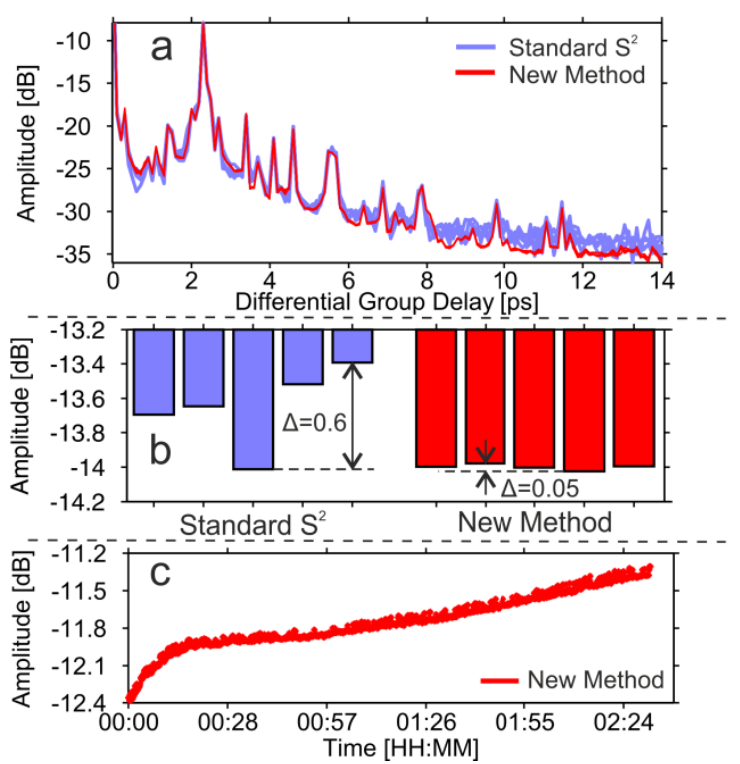

Fig. 2: Comparison between conventional (blue) and new (res) method (5 repeat measurements): (a) DGD graphs and (b) corresponding MPI against measurement start time. Note excellent consistency of new method. (c) Long term monitoring of small variations of MPI of TMF over time (924 measurements over $2.5 \mathrm{hrs}$ ).

in 22 seconds $^{5}$ (4.5 frames/s). The time per measurement is predominantly determined by the need to command the TLS to tune to and settle at a new wavelength each frame. This limitation is removed in our method; instead the TLS is rapidly scanned across the full measurement bandwidth with a single command and the CCD is triggered to record images at set time intervals, based on the desired resolution, which captures an image, Fig. 1b (red). The only drawback is that scanning the laser increases the effective linewidth of the laser (from $0.8 \mathrm{fm}$ to $0.5 \mathrm{pm}$ [due to the $1 \mathrm{~ms}$ integration time of our CCD]). Yet, this is still smaller than the TLS wavelength resolution $(1 \mathrm{pm})$, so does not affect the maximum DGD value. To date we have achieved data capture of 500 frames in one second, giving a $110 x$ increase in wavelength capture rate over the current state of the art for a single-shot measurement. With this change in method, we have developed a system allowing near real time monitoring of the FUT. It has the ability to process the DGD graph in $0.8 \mathrm{~s}$ with a $7 \mathrm{~s}$ wait between taking measurements. The main limitation on the repetition rate is calling the TLS to start the scan each time. This can be dramatically reduced to $<0.3 \mathrm{~s}$ by cycling the TLS. Furthermore, the wait time could be removed by synchronizing another scanning laser, allowing the method to produce real-time results. Without inline data processing we have obtained up to 20,000 wavelengths in a single sweep, (total time 40s), giving the possibility of a practical single-shot, high-resolution spectrogram. Our faster system has many advantages compared to a typical $S^{2}$ implementation such as: higher total frame number per unit measurement time, reduced temporal noise and increased measurement rate. The reduced test time opens a new array of possible measurements, e.g. monitoring the manufacturing of MM devices.

To illustrate the effect that increasing the measurement speed has on the DGD graph, our fiber mode characterization technique was used to characterize a two mode fiber ${ }^{6}$ (TMF). The measurement range was $1545-1565 \mathrm{~nm}$ with $40 \mathrm{pm}$ resolution. Light was launched into the TMF by butt-coupling from a standard single mode fiber (SMF). The mode field diameter (MFD) mismatch $(T M F=16.4 \mu \mathrm{m}, \mathrm{SMF}=10.5 \mu \mathrm{m})$ enabled simultaneous excitation of both $\mathrm{LP}_{01}$ and $\mathrm{LP}_{11}$ modes. The modal coupling was controllable and monitoring was done in near real-time, as made possible by the speed improvement offered by our new technique. Five readings were taken with the standard $S^{2}$ method (1 wavelength every $1.5 \mathrm{~s}$ ), and compared with five readings from the new method ( 1 wavelength every $0.02 \mathrm{~s}$ ), Fig. $2 \mathrm{a}$. The new method shows, Fig. $2 b$, a reduced noise floor and highly repeatable results ( $\triangle \mathrm{MPI}$ $\sim 0.05 \mathrm{~dB}$ ) compared to the conventional $\mathrm{S}^{2}$ method ( $\triangle \mathrm{MPI} 0.6 \mathrm{~dB})$. To further illustrate the point the new method was used to monitor the MPI over 2.5 hours (924 MPI values); Fig.2c shows a slow MPI drift $(<1 \mathrm{~dB})$ likely due to environmental factors, which is only visible with the MPI stability offered by the new method.

We note however that the number of peaks observed in Fig. 1a is unexpected and would appear to be inconsistent with a TMF. In fact, we demonstrated that both measurements are affected by spurious interference peaks.

\section{Identifying the source of spurious peaks}

Four lengths of step-index, TMF were measured, with lengths $2,4,10$ and $22 \mathrm{~m}$, using the new method (501 wavelengths centered at $1550 \mathrm{~nm})$. The spectral resolution and bandwidth varied from $6-80 \mathrm{pm}$ and $3-40 \mathrm{~nm}$ respectively, to ensure the normalized differential group resolution $(0.1 \mathrm{ps} / \mathrm{m})$ was kept constant, which verified the $D G D$ of the $L P_{01}-L P_{11}$ modes to be $2.3 \mathrm{ps} / \mathrm{m}$ in all measurements. To distinguish between spurious peaks and useful data we removed the fiber length normalization on the DGD, Fig. 3a; clearly many interference peaks occurring from 5.7-50ps show the same DGD regardless of the fiber length.

To establish the origin of the spurious peaks we carried out two measurements with a simplified configuration; firstly with the FUT 

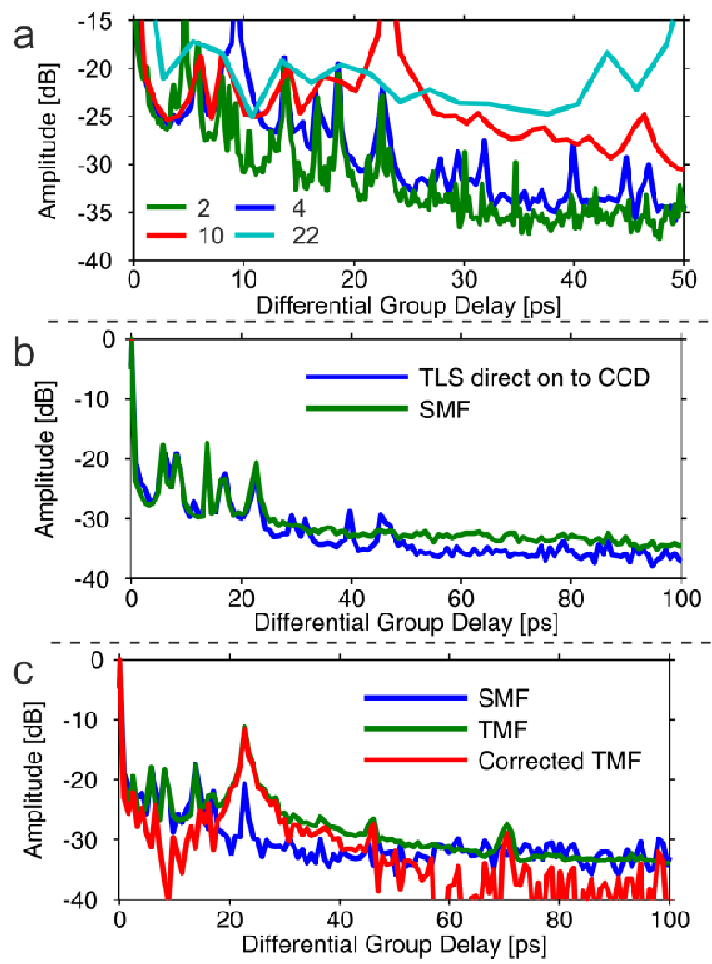

Fig. 3 DGD of; a) 2, 4, 10 and 22m lengths of TMF, b) TLS directly onto CCD \& SMF as FUT, c) Corrected TMF as FUT

changed to SMF and secondly, a direct measurement from the TLS to the CCD without a fiber, Fig. 3b. Both measurements had a 1550$1560 \mathrm{~nm}$ bandwidth and a $20 \mathrm{pm}$ resolution, giving a 0.82 ps delay resolution. Peaks at $4.6,8$, $13.7,16.9,22.6 p s \pm 0.4 p s$ are common to both measurements and correspond to double reflections from the CCD. Further reflection peaks at $32,40.2,45$ and $46.7 p s \pm 0.4 p s$ were found to be due to reflections from the microscope objective lens used for magnification. Other peaks at higher DGD values have also been measured and correspond to multiple double reflections from the previously mentioned sources.

More generally, double reflections can occur within and between any optics in the system. For a TLS with maximum resolution of $1 \mathrm{pm}$, this means reflections from gaps of up to $600 \mathrm{~mm}$ in air and $400 \mathrm{~mm}$ for glass with $n=1.5$ can be detected. Given the dispersive nature of most optical components, the delay of these systematic spurious peaks will change as a function of wavelength and therefore they must be characterized using a spectrogram approach.

If unaccounted for, spurious interference peaks of the $\mathrm{LP}_{01}$ modes will limit the useable parameters for a given measurement, in this case DGDs over 50ps. For MM fibers, each guided mode could be reflected from the same sources, leading to possible incorrect identification of the true modal interference peaks, or overlap between spurious and real peaks. This could push up and/or widen the interference peaks and distort the intensity and phase images. If the DGD resolution is coarse, as is the case for typical $S^{2}$ results, Fig. 3a $(22 \mathrm{~m})$, the reflection peaks will not be properly resolved thus degrading the measurement noise floor, in this case by $\sim 10 \mathrm{~dB}$; this could wrongly be attributed to distributed scattering between the $L P_{01}$ and $L P_{11}$ modes.

Removing the spurious peaks can be achieved by changing components and/or applying better anti-reflection coatings. However, this is not always possible. Another method is to numerically remove the systematic peaks, as they will appear at the same relative time and with the same magnitude in each measurement. This is shown using a TMF, Fig. 3c, where the DGD measurement of a SMF is subtracted from the FUT results to produce a corrected DGD estimate.

\section{Conclusions}

We have developed a fiber modal characterization method based on the $S^{2}$ technique that can take a measurement with 501 wavelengths in 1s. This, alongside inline data processing, opens up the prospect of near real time modal characterization. With this, system, we have studied and removed the systematic spurious double reflection peaks present in our measured DGD data thereby improving the quality and reliability of our results.

\section{Acknowledgements}

This work was supported by the European Communities $7^{\text {th }}$ Framework Programme under grant agreement 228033 (MODE-GAP).

\section{References}

[1] J. W. Nicholson et al., "Measuring the Modal Content of Large-Mode-Area Fibers" IEEE J. Sel. Top. Quant. Elect., Vol. 15, no. 61 (2009).

[2] A.M. DeSantolo et al., "High Resolution $S^{2}$ Mode Imaging of Photonic Bandgap Fiber" Proc. OSA/ CLEO, CFM4, Baltimore (2011)

[3] J.W. Nicholson et.al. "Measuring higher-order modes in a low-loss hollow-core, photonic-bandgap fiber" Opt. Exp. Vol. 20, no.18 (2012)

[4] D. M. Nguyen et al,. "Modal Decomposition Technique for Multimode Fibers" App. Lett., Vol. 51, no. 4 (2012)

[5] L. Grüner-Nielsen et al,. "Stable Mode Converter for Conversion between LP01 and LP11 Using a Thermally Induced Long Period Grating" Proc. IEEE Sum. Top. WC1.2, Washington (2012)

[6] L. Grüner-Nielsen et al,. "Measuring Distributed Mode Scattering in Few Mode Fibers with high and low Differential Group Delay" Proc. IEEE Sum. Top. TuC1.3, Hawaii (2013) 